Regards sur l'économie allemande

Bulletin économique du CIRAC

82 | 2007

Varia

\title{
La politique énergétique sous la présidence allemande de l'UE
}

Florence Autret

\section{(2) OpenEdition}

\section{Journals}

Édition électronique

URL : http://journals.openedition.org/rea/632

DOI : $10.4000 /$ rea.632

ISBN : 978-2-8218-0860-7

ISSN : 1965-0787

\section{Éditeur}

CIRAC

\section{Édition imprimée}

Date de publication : 1 juillet 2007

Pagination : 13-18

ISSN : 1156-8992

\section{Référence électronique}

Florence Autret, « La politique énergétique sous la présidence allemande de l'UE », Regards sur l'économie allemande [En ligne], 82 I juillet 2007, document 2, mis en ligne le 01 juillet 2009, consulté le 22 avril 2019. URL : http://journals.openedition.org/rea/632 ; DOI : 10.4000/rea.632 


\title{
La politique énergétique sous la présidence allemande de 1'UE
}

\author{
Florence Autret
}

\begin{abstract}
L'Allemagne a placé la question énergétique au centre de sa présidence de l'Union européenne et du groupe des huit pays les plus industrialisés. Ce choix s'explique par les difficultés rencontrées par la grande coalition dans la recherche du compromis entre compétitivité, durabilité environnementale et sécurité des approvisionnements qui est au cœur de toute politique énergétique. Effectivement, la chancelière allemande a réussi à mettre habilement au service de ses propres contraintes politiques le levier européen, tout en apportant à l'Union un surcroît de cohérence et de crédibilité dans ce domaine.
\end{abstract}

\section{La difficile équation énergétique nationale}

Depuis une dizaine d'années, la politique énergétique allemande traverse une zone de fortes turbulences sous l'effet de facteurs internes et externes. Dans un premier temps, la libéralisation des marchés de l'énergie introduite progressivement depuis la fin des années 1990 a initié un mouvement de concentration qui a profondément transformé la structure de l'offre et renforcé la position d'une poignée de très grands acteurs. En 2000 est ensuite venue la décision d'abandonner à moyen terme la production d'électricité nucléaire, décision qui appelle une transformation de la structure de l'approvisionnement en énergie. Enfin, la croissance des pays émergents et l'instabilité politique dans les régions de production de pétrole et de gaz se sont traduites par un nouveau " choc pétrolier ", en fait pétrolier et gazier, qui n'a rien de ponctuel, les prix continuant à augmenter. A cela s'ajoute la montée du problème du changement climatique en tête de l'agenda politique européen et mondial. Tous ces facteurs ont profondément transformé la situation énergétique de l'Allemagne et complexifié le jeu des contraintes qui s'exercent sur ses autorités politiques.

L'Allemagne a un triple problème, de prix, de régulation et de sécurité. Comme tous les autres pays européens, elle assiste à une augmentation tendancielle des coûts de son approvisionnement en énergie, et notablement en électricité. Pour les industriels, entre 1990 et 2006, le prix du kWh a augmenté de $65 \%$ et celui du gaz naturel a été multiplié par plus de deux. A titre d'exemple, pour la seule année 2006, le prix du $\mathrm{kWh}$ moyen pour les ménages a augmenté de $2,6 \%$ par rapport à $2005(+4,6 \%$ pour l'Europe des 25$)$, alors que les prix pour l'industrie (en moyenne) faisaient un bond de $10 \%$ (UE $25:+15,5 \%$, source Eurostat). Concernant les prix du gaz naturel, cette progression s'explique par la flambée des cours mondiaux. Pour ce qui est de l'électricité, elle résulte de plusieurs facteurs : le recul de la production nucléaire qui se faisait à un coût marginal limité (le parc étant à maturité), l'augmentation de la part des centrales au gaz (donc indirectement celui du prix de la ressource) mais aussi, quoiqu'il soit plus difficile de mesurer ce facteur, la dérégulation du marché qui a eu des effets pervers sur la concurrence.

Depuis la première loi de libéralisation de 1998, qui a été suivie par une deuxième en 2005 , le gouvernement fédéral n'en finit pas de redéfinir le cadre réglementaire de cette activité. L'Allemagne ayant fait le double choix d'assurer un accès négocié des tiers à ses réseaux électrique et gazier, d'une part, et de

Augmentation des prix

Une régulation en chantier 
L'insécurité des approvisionnements

La difficile question du nucléaire

Le problème de la dépendance ne pas avoir imposé la séparation légale des activités de transport, de production et de distribution, d'autre part, ses autorités sont confrontées à un travail de régulation colossal. Dans le contexte d'une relative pénurie de l'offre, la tentation de tirer une rente de leur position est grande pour les opérateurs, producteurs et/ou exploitants de réseau. Michael Glos, le ministre fédéral de l'Economie a dû s'y reprendre à plusieurs reprises pour faire adopter par le gouvernement fédéral (décision du 13/6/2007) un décret qui impose aux opérateurs une baisse progressive de leurs tarifs de transport (lesquels représentent pour l'électricité un tiers du prix facturé à l'utilisateur final) et les oblige à augmenter la productivité de leurs installations tout en encourageant les nouveaux investissements (Kraftwerks-Netzanschluss-Verordnung). Ce décret n'est pas encore entré en application, faute d'avoir été approuvé par la chambre des Länder, le Bundesrat. Le gouvernement a également engagé une nouvelle modification de la loi sur les entraves à la concurrence (Gesetz gegen Wettbewerbsbeschränkungen, GWB) afin de faciliter l'activité des autorités anticartels fédérales et régionales. Jusqu'à présent celles-ci se sont montrées impuissantes à empêcher les phénomènes de rente ou les abus de marché.

L'Allemagne fait également face à un problème de sécurité de son approvisionnement, qui lui-même comporte deux aspects, interne et externe. Au plan interne, les capacités de transport et de production ne sont pas garanties à terme. Le 4 novembre 2006, l'Allemagne a connu son premier black-out dû à une insuffisance du réseau d'E.On. Le bilan de cet incident publié par la Commission européenne est accablant pour le gestionnaire de réseau (IP/07/100, 30-01-2007, «principaux enseignements à tirer de la panne d'électricité de novembre $2006 »)$. Bruxelles met plus généralement en cause «l'insuffisance des investissements dans le réseau, au niveau tant de sa fiabilité que de son exploitation ». Berlin doit en outre gérer les conséquences, sur ses capacités de production, de la décision de sortie du nucléaire, par fermeture successive des centrales en activité, d'ici 2022. Jusqu'à présent, seules deux centrales ont été fermées. Mais au total, ce sont $20 \mathrm{MW}$ (27\% des capacités installées) qui devront être remplacés. Pour l'instant, la part du nucléaire dans la production électrique est restée relativement stable (17\%) mais elle est appelée à baisser significativement et, en dépit des efforts réalisés pour développer les énergies renouvelables, la principale alternative reste la construction de centrales au gaz.

A la tête d'une grande coalition, la chancelière chrétienne-démocrate Angela Merkel est liée par l'accord d'abandon du nucléaire signé en 2000 par son prédécesseur social-démocrate Gerhard Schröder. Cependant, elle cherche à retrouver des marges de manœuvre sur ce sujet. Depuis le grand «Sommet de l'énergie » (Energiegipfel) d'avril 2006, l'idée qu'on puisse rediscuter de ce choix n'est plus taboue. Mais le gouvernement fédéral tient à inscrire cette décision dans le cadre de la réflexion plus générale sur la politique énergétique. L'Energiegipfel, qui réunit l'ensemble des opérateurs économiques et des responsables politiques, et a siégé à nouveau à l'automne 2006, doit précisément permettre d'en jeter les bases «avant fin 2007 », selon le ministère fédéral de l'Economie. Le temps presse : quatre nouvelles tranches doivent être retirées du réseau d'ici 2009 en vertu du calendrier arrêté en 2000.

Dernière difficulté : la dépendance énergétique. C'est un fait récurrent dans tous les pays européens à l'exception du Danemark et de la Norvège (non membre de l'Union européenne). Mais cette dépendance est encore accrue en République fédérale par le poids des sources d'énergie fossile dans la structure de l'approvisionnement. L'Allemagne importe $97 \%$ de son pétrole, $83 \%$ de son gaz, $61 \%$ de son charbon et la totalité de l'uranium. Sa dépendance est particulièrement forte vis-à-vis de la Communauté des Etats Indépendants (Russie, Biélorussie et républiques d'Asie centrale) qui lui livre $41 \%$ du pétrole, $37 \%$ du gaz, $19 \%$ de l'uranium et $8 \%$ du charbon qu'elle consomme. Voilà qui suffirait à faire de l'Allemagne et de la Russie des partenaires stratégiques l'un 
de l'autre. "Pendant la Guerre froide, la Russie a approvisionné de façon fiable la République fédérale ", rassurait la chancelière récemment. Certes, Moscou a aussi besoin de Berlin, mais la dépendance croissante de la seconde vis-à-vis de la première ne laisse pas d'inquiéter. Tel est le contexte dans lequel s'inscrit l'élaboration par les autorités allemandes de l'agenda de leur présidence semestrielle de l'Union européenne qui s'est terminée le 31 juin 2007.

\section{Les enjeux de la présidence allemande de l'UE}

La plupart des autres pays européens faisant face aux mêmes dilemmes et difficultés dans ce domaine, la politique énergétique a d'emblée été pour Berlin un objectif prioritaire de sa présidence, à côté des négociations sur les institutions de l'Union européenne et de l'approfondissement des relations transatlantiques. En élargissant la focale, deux sujets majeurs ont émergé, qui soustendent les priorités allemandes : premièrement, les suites de la libéralisation des marchés qui, dix ans après son lancement, apparaît déséquilibrée; deuxièmement, les risques qui pèsent sur l'approvisionnement énergétique du continent.

Bien que deux des trois traités d'origine de l'Union européenne aient porté sur l'énergie, la politique énergétique n'est pas une compétence communautaire. Les conditions historiques et politiques d'organisation des marchés sont profondément différentes d'un pays à l'autre et expliquent une grande diversité des modes d'organisation et de la propriété des capacités de transport, de production ou de distribution.

Pourtant, l'intervention de l'Union européenne a profondément transformé les marchés énergétiques. Mais, à défaut de compétences proprement communautaires, elle s'est faite sous couvert de la politique de la concurrence. Résultat, par propension autant que par nécessité, la Commission européenne a séparé dans son approche la question des investissements, laissée aux Etats membres, de celle de la concurrence et des prix, qui relève bien d'un cadre réglementaire commun. Comme l'écrit Jan-Horst Keppler, "la politique européenne de l'énergie a été conçue jusqu'à récemment comme un appendice d'autres politiques : libéralisation et réalisation du marché unique, politique de protection de l'environnement ». Les augmentations importantes des prix partout en Europe ces dernières années (alors que la Commission promettait une baisse des tarifs sous l'effet de la libéralisation) et les problèmes de sécurisation à terme des capacités de production manifestent, sinon l'échec, du moins les insuffisances de l'approche communautaire. La situation allemande est exemplaire à cet égard. Ajoutée à la décision de sortie du nucléaire, la libéralisation et l'incertitude sur les futurs retours sur investissement qu'elle crée mécaniquement ont encouragé un recours massif aux centrales à cycle combiné utilisant le gaz naturel, la technologie de loin la plus performante en termes de flexibilité et de retour sur investissement.

Le second écueil de la politique énergétique européenne, qui n'est pas sans lien avec le premier, réside dans la dépendance énergétique de l'UE vis-à-vis de ses fournisseurs extérieurs, ceci dans un climat de grande incertitude internationale. La libéralisation a en effet accru cette dépendance en créant un effet d'aubaine au bénéfice du gaz naturel. Actuellement la quantité de gaz consommé par unité de richesse produite (intensité énergétique) progresse, alors que pour l'ensemble des énergies, elle diminue tendanciellement dans les pays industrialisés, sous l'effet du progrès technologique. Cette dépendance ne serait pas problématique si elle ne s'inscrivait dans un contexte de forte instabilité géopolitique. Là encore, la Commission européenne commence à peine à prendre la mesure des enjeux, mais de façon hésitante, compte tenu de son organisation interne et de ses compétences. Le président de la Commission européenne, José Manuel Barroso, a annoncé l'an dernier qu'il mettrait la

\section{Une libéralisation déséquilibrée}

La politique énergétique n'est pas une compétence communautaire

Mais elle s'effectue " comme un appendice d'autres politiques "

Dépendance énergétique et instabilité géopolitique 
Le manque de cohérence communautaire

La difficile coopération entre UE et Etats membres

Eclatement des compétences à la Commission question énergétique au centre de tous les sommets que l'Union européenne tiendrait avec des pays tiers en 2007, à commencer évidemment par la Russie. La Russie est liée à l'Union européenne par un accord de partenariat et de coopération vieux de 10 ans mais a refusé de ratifier la Charte de l'énergie, dont l'Europe tente, jusqu'à présent en vain, de faire inscrire les principes dans cet accord bilatéral. En réalité, l'Europe est loin d'avoir stabilisé sa diplomatie énergétique vis-à-vis de la Russie. Elle subit de plein fouet les pressions des autorités politiques fédérales russes qui opèrent la renationalisation des capacités de production du pays et les ambitions de l'exploitant public des ressources en gaz Gazprom qui utilise sa position de fournisseur pour tenter d'entrer sur le marché de la distribution en Europe. Les relations entre Gazprom et les opérateurs européens sont ainsi émaillées de renégociations comme récemment dans le cas de BP au sujet de l'exploitation du gisement de Kovykta.

Dans ce contexte, le travail de la présidence allemande sur la question énergétique se lit comme une tentative de donner à la politique européenne la cohérence et la force qui lui font défaut. Mais cette ambition se heurte d'emblée à deux difficultés. Le fait que la politique énergétique européenne reste une politique de la concurrence, laquelle est un domaine de compétence jalousement gardé par la Commission européenne, réduit la marge de manœuvre des Etats membres. Alors même que le gouvernement allemand, au nom de la présidence de l'Union, déroulait sa proposition de plan d'action européen, la Commission européenne pointait indirectement du doigt les manquements de l'Allemagne aux règles de concurrence. Les attaques de la Commissaire à la concurrence, Nelly Kroes, contre l'intégration verticale de certains grands opérateurs, dont E.On ou EDF, n'ont jamais été aussi vives que pendant l'hiver 2007, N. Kroes allant jusqu'à menacer d'imposer une séparation légale des activités, autrement dit un démantèlement des sociétés concernées.

La seconde difficulté résulte du partage des compétences entre l'Union et ses Etats membres. Berlin n'a jamais laissé penser que la solution viendrait d'une communautarisation de la politique énergétique, au sens d'une plus grande délégation de pouvoirs aux institutions communes. Elle a même répété à de nombreuses reprises que cette politique restait une compétence nationale. Mais force est de constater qu'il existe un partage des tâches de facto. Le travail de mise en cohérence nécessaire pour concilier les contraintes de prix, de régulation et de sécurité, doit s'opérer dans le cadre de ce partage, lequel résulte d'un effet d'opportunité politique induit par la compétence communautaire dans le domaine de la concurrence et de la volonté d'affirmation de Bruxelles, plus que d'un acte délibéré des Etats membres au sein de l'Union européenne. Dans ces conditions, la coordination entre politiques européenne et nationale est une gageure puisqu'elle doit se construire sur la base d'une compétition objective entre les deux niveaux de pouvoir.

Dans sa tentative de prendre en compte les multiples dimensions de la politique de l'énergie, la Commission européenne juxtapose les initiatives plutôt qu'elle ne les organise selon un plan cohérent. II est frappant de noter le nombre de commissaires européens qui traitent du sujet. Charlie McCreevy, en charge du marché intérieur, est en première ligne (la libéralisation des marchés de l'électricité et du gaz est une des dimensions de l'achèvement du marché intérieur). Nelly Kroes intervient sur tous les sujets de concurrence : contrôle des concentrations dans le secteur (comme le rapprochement avorté E.On/Endesa ou potentiel GDF/Suez), mais aussi contrôle d'éventuels abus de position dominante des exploitants de réseau ou encore d'accords de marché. Andris Piebalgs, en charge du portefeuille de l'énergie, est actif essentiellement sur les infrastructures et le dialogue avec les pays tiers, un domaine où est également présente Benita Ferroro-Waldner, en charge des relations extérieures. Enfin, tous les aspects environnementaux (système européen d'échange de droits d'émission de CO2 et énergies renouvelables) sont du ressort de Stavros Dimas, Commissaire à l'environnement. A quoi s'ajoute la contribution du Haut Représentant de 
I'UE, Javier Solana, sur la politique énergétique extérieure de l'UE publiée en 2006. En dépit de cet éclatement des initiatives, le président Barroso avait mis sur la table en janvier 2007 un "paquet énergie ", compilation foisonnante, qui fut la contribution de l'exécutif communautaire au Sommet de printemps.

Les objectifs de la présidence allemande de l'UE, au nombre de six, n'ont pas échappé à ce foisonnement. Ils comprenaient l'intensification des relations extérieures, l'intensification du travail sur des perspectives de long terme de l'approvisionnement et de la demande, l'amélioration du fonctionnement du marché unique de l'électricité et du gaz, l'amélioration de l'efficience énergétique, grâce à l'innovation (qui est aussi un moyen de réduire la dépendance), l'approfondissement du travail sur les énergies renouvelables et, enfin, la formulation d'une position européenne sur le changement climatique après 2012, horizon du Protocole de Kyoto pour la réduction des émissions de gaz à effets de serre.

L'Allemagne avait donc placé la barre très haut mais elle disposait d'atouts réels pour réussir, tels que sa relation privilégiée avec la Russie, qui repose sur des partenariats industriels (en capital ou contractuels), de sa position centrale sur le marché électrique qui en fait un maillon essentiel de la chaîne d'approvisionnement et de transport du gaz comme de l'électricité, et enfin de sa position de leader dans le domaine des technologies environnementales (énergies renouvelables ou efficience de la production conventionnelle).

\section{Des gages pour l'avenir plus que des résultats immédiats}

Le résultat de sa présidence n'est peut-être pas à la hauteur des enjeux actuels, mais il est remarquable compte tenu des difficultés qu'il fallait surmonter. Au Conseil européen des 8 et 9 mars, la chancelière fédérale a réussi à faire adopter par ses homologues, au prix de changements mineurs, son plan d'action "Une politique énergétique pour l'Europe ", minutieusement élaboré dès 2006. Ce document de 7 pages annexé aux conclusions du Conseil constitue désormais - plus que les stratégies successives proposées par la Commission européenne - une véritable feuille de route à la fois pour les Etats membres et pour la Commission.

L'objectif ambitieux de -20\% à l'échéance de 2020 en dessous de leur niveau de 1990 , qui aurait pu être porté à $-30 \%$ en cas d'engagement réciproque des autres pays industrialisés, a été assorti d'objectifs secondaires : $+20 \%$ d'énergies renouvelables, $+20 \%$ d'économies d'énergie et $+10 \%$ de biocarburants. Cette stratégie comporte des risques, particulièrement celui d'un désavantage compétitif pour les producteurs européens (voir les lamentations de certains industriels au sujet du système d'échange de droits à polluer). La chancelière, qui assumait en même temps que la présidence de l'Union celle du groupe des huit nations les plus industrialisées (G8) n'a pas réussi à rallier les Etats-Unis au principe d'un engagement chiffré comparable à celui de l'Europe lors du Sommet de Heiligendamm. Par son engagement unilatéral, toutefois, l'Europe affirme son rôle de précurseur en matière de réduction des émissions.

Ce sujet a alimenté la dramaturgie habituelle des sommets de chefs d'Etat avec son lot de suspens sur l'issue des négociations, il a capté toute l'attention des médias et permis aux dirigeants européens d'encaisser les gains politiques d'un compromis dans un contexte de montée des peurs face aux conséquences du changement climatique. Mais c'est au moins autant en termes de sécurité d'approvisionnement qu'il faut mesurer les retombées possibles de la discipline écologique que les Européens ont décidé de s'imposer. La stratégie européenne sur le climat va d'abord continuer à orienter l'effort de recherche et d'investissement vers les sources d'énergie alternative. Elle va ensuite améliorer la compétitivité tant des renouvelables que du nucléaire, lesquels limitent la dépendance. Si le plan d'action de mars 2007 réaffirme l'autonomie complète des Etats membres quant au recours, ou non, à l'énergie nucléaire, il préconise
Les atouts de l'Allemagne

Rôle précurseur pour la réduction des émissions 
Une politique de régulation plus fine également de traiter au niveau européen les problèmes de sûreté et de gestion des déchets (création d'un groupe de haut niveau).

En matière de régulation, il confirme le virage pris par la Commission européenne d'accompagner la politique de libéralisation d'une véritable stratégie sur les infrastructures de transport. Bruxelles est en train de se défaire de la relative naïveté qui avait présidée aux premières étapes de la libéralisation et qui voulait qu'une concurrence accrue soit optimale aussi bien en termes de prix, donc de compétitivité, que d'investissements. La réflexion qui s'amorce fait une place à la structure, régionale, des marchés, à l'organisation de la régulation ( $\mathrm{y}$ compris la coordination entre régulateurs européens) et finalement aux dilemmes inhérents à toute politique énergétique: entre concurrence et sécurité, entre sécurité et environnement, et enfin entre concurrence et environnement. Les pressions exercées par Gazprom pour accéder à l'aval du marché européen et la peur de dumping qu'elles font naître ne sont pas étrangères à cet accès de réalisme. Les 27 invitent ainsi la Commission européenne à "évaluer l'impact sur le marché intérieur des compagnies énergétiques verticalement intégrées originaires de pays tiers et à déterminer comment mettre en œuvre le principe de réciprocité ».

Au REGARD DE CES RESULTATS, peut-on dire que la présidence allemande de I'UE a contribué à sortir le gouvernement fédéral des impasses actuelles de sa politique énergétique ? La réponse est globalement positive. La stratégie européenne qui se dessine, tout en faisant de l'Europe un précurseur, valorise les atouts technologiques allemands. Par ailleurs, elle souligne l'urgence de la question nucléaire et pourrait faciliter la recherche d'un nouveau compromis sur ce terrain à Berlin. Elle confirme enfin la position clef de l'Allemagne dans les discussions avec la Russie tout en consolidant une approche multilatérale qui renforce la position des acheteurs européens. Ajoutons enfin que le déblocage probable du débat institutionnel européen qui est aussi largement à mettre au crédit de Berlin ne pourra que faciliter la mise en œuvre du plan d'action adopté en mars.

\section{Indications bibliographiques}

CONSEIL DE L'UE, Plan d'action du Conseil européen (2007-2009) - Une politique énergétique pour l'Europe, Annexe 1 au document : Conseil européen de Bruxelles, 8-9 mars 2007, Conclusions de la présidence, Bruxelles, 02-05-07, Réf. 7224/1/07 REV 1 CONCL 1

KEPPLER J.-H., « La sécurité des approvisionnements énergétiques en Europe : principes et mesures ", Notes de l'Ifri, Institut français des relations internationales, Paris, avril 2007

PaillaRd C.-A., «Les défis énergétiques lancés à l'Europe », in CHOPIN T ., Foucher M. (dir.), L'Etat de l'Union 2007, Rapport Schuman sur l'Europe, Paris, 2007

Röller L.-H., Delgado J., Friederiszick H.-W., Energy : Choices for Europe, Bruegel Blueprint Serie, 2007. 